\title{
Conessine as a novel inhibitor of multidrug efflux pump systems in Pseudomonas aeruginosa
}

Thanyaluck Siriyong ${ }^{1}$, Potjanee Srimanote ${ }^{2}$, Sasitorn Chusri ${ }^{3}$, Boon-ek Yingyongnarongkul ${ }^{4}$, Channarong Suaisom ${ }^{4}$, Varomyalin Tipmanee ${ }^{5}$ and Supayang Piyawan Voravuthikunchai ${ }^{*^{*}}$

\begin{abstract}
Background: Holarrhena antidysenterica has been employed as an ethnobotanical plant for the treatment of dysentery, diarrhoea, fever, and bacterial infections. Biological activities of the principle compound, conessine including anti-diarrhoea and anti-plasmodial effects were documented. Our previous study reported potency of Holarrhena antidysenterica extract and conessine as resistance modifying agents against extensively drug-resistant Acinetobacter baumannii. This study aimed to investigate (i) whether conessine, a steroidal alkaloid compound, could act as a resistance modifying agent against multidrug-resistant Pseudomonas aeruginosa, and (ii) whether MexAB-OprM efflux pump involved in the mechanism.
\end{abstract}

Methods: Conessine combined with various antibiotics were determined for synergistic activity against $P$. aeruginosa PAO1 strain K767 (wild-type), K1455 (MexAB-OprM overexpressed), and K1523 (MexB deletion). H33342 accumulation assay was used to evaluate efflux pump inhibition while NPN uptake assay was assessed membrane permeabilization.

Results: Conessine significantly reduced MICs of all antibiotics by at least 8-fold in MexAB-OprM overexpressed strain. The levels were comparable to those obtained in wild-type strain for cefotaxime, levofloxacin, and tetracycline. With erythromycin, novobiocin, and rifampicin, MICs were 4- to 8-fold less than MICs of the wild-type strain. Loss of MexAB-OprM due to deletion of mexB affected susceptibility to almost all antibiotics, except novobiocin. Synergistic activities between other antibiotics (except novobiocin) and conessine observed in MexB deletion strain suggested that conessine might inhibit other efflux systems present in $P$. aeruginosa. Inhibition of H33342 efflux in the tested strains clearly demonstrated that conessine inhibited MexAB-OprM pump. In contrast, the mode of action as a membrane permeabilizer was not observed after treatment with conessine as evidenced by no accumulation of 1-N-phenylnaphthylamine.

Conclusions: The results suggested that conessine could be applied as a novel efflux pump inhibitor to restore antibiotic activity by inhibiting efflux pump systems in $P$. aeruginosa. The findings speculated that conessine may also have a potential to be active against homologous resistance-nodulation-division (RND) family in other Gramnegative pathogens.

Keywords: Conessine, Efflux pump inhibitor, MexAB-OprM efflux system, Pseudomonas aeruginosa

\footnotetext{
* Correspondence: supayang.v@psu.ac.th

${ }^{1}$ Department of Microbiology and Excellence Research Laboratory on Natural

Products, Faculty of Science and Natural Product Research Center of

Excellence, Prince of Songkla University, Hat Yai, Songkhla 90112, Thailand

Full list of author information is available at the end of the article
}

(c) The Author(s). 2017 Open Access This article is distributed under the terms of the Creative Commons Attribution 4.0 International License (http://creativecommons.org/licenses/by/4.0/), which permits unrestricted use, distribution, and reproduction in any medium, provided you give appropriate credit to the original author(s) and the source, provide a link to the Creative Commons license, and indicate if changes were made. The Creative Commons Public Domain Dedication waiver (http://creativecommons.org/publicdomain/zero/1.0/) applies to the data made available in this article, unless otherwise stated. 


\section{Background}

Pseudomonas aeruginosa is an emerging global opportunistic multidrug-resistant (MDR) pathogen associated with high morbidity and mortality rates. The organism causes a number of infections such as pneumonia, urinary tract infection, and sepsis [1]. Broad spectrum antimicrobial resistance in MDR $P$. aeruginosa seriously limits effective therapeutic options. MDR phenotype can be mediated by a variety of resistance mechanisms including chromosomally encoded enzymes, expression of efflux pumps, and low membrane permeability. Various chromosomally encoded efflux systems and outer membrane porins have been identified as important contributors to resistance [1]. The most relevant multidrug efflux systems in MDR pathogens are members of resistance-nodulation-division (RND) family. A number of MDR RND efflux pumps have been characterized in clinical isolates of $P$. aeruginosa, namely MexAB-OprM, MexCD-OprJ, MexEF-OprN, and MexXY-OprM. Among these pumps, only MexAB-OprM is constitutively expressed at a level sufficient to confer intrinsic MDR in wild-type $P$. aeruginosa strains [2]. MexAB-OprM transports a number of antibiotics including fluoroquinolones, $\beta$-lactams, tetracycline, macrolides, chloramphenicol, novobiocin, trimethoprim, and sulphonamides [3]. Mutations in nalB or mexR resulted in overexpression of MexAB-OprM efflux pump [4].

Combination therapy may be beneficial for controlling MDR P. aeruginosa that could restore susceptibility to various antibiotics [5-7]. A number of potent efflux pump inhibitors including phenylalanyl arginyl $\beta$-naphthylamide $(\mathrm{PA} \beta \mathrm{N})$, carbonyl cyanide $m$-chlorophenylhydrazone (CCCP), quinoline derivatives, and 1-(1-Naphthylmethyl)-piperazine (NMP) have been reported to enhance antibiotic activity against antibioticresistant Gram-negative bacteria. In addition, various compounds such as $\mathrm{PA} \beta \mathrm{N}$, ethylenediaminetetraacetic acid (EDTA), and polymyxin B nonapeptide (PMBN) have been documented to permeabilize the bacterial outer membrane. However, none has reached potential clinical applications because of its toxicity [8].

A number of plant extracts and phytochemical products have demonstrated their potential as synergists or potentiators of other antibacterial agents [9]. Curcumin derived from Curcuma longa inhibited efflux pump systems in $P$. aeruginosa, resulting in restoring gentamicin and ciprofloxacin activity [10]. Extract from Holarrhena antidysenterica displayed resistance modifying ability to enhance novobiocin and rifampicin activity against Acinetobacter baumannii $[11,12]$. It has been demonstrated that the extract potentiated the effect of antibiotics by acting as a permeabilizer [13]. Moreover, a recent study indicated that both Holarrhena antidysenterica extract and conessine, a steroidal alkaloid compound, could restore antibiotic activity due to interference with AdeIJK pump in A. baumannii [14]. Previous study documented that AdeIJK pump and MexAB-OprM pump are functionally equivalent pumps in both organisms [15].

Holarrhena antidysenterica belonging to family Apocynaceae has been employed as an ethnobotanical plant for the treatment of dysentery, diarrhoea, fever, and bacterial infections. Biological activities of the plant including antimalarial, anti-diabetic, anti-oxidant, antiurolithic, anti-mutagenic, CNS-stimulating, angiotensinconverting-enzyme inhibitory, and acetylcholinesterase inhibitory activity were documented [16]. In contrast, anti-diarrhoea and anti-plasmodial effects of conessine were briefly mentioned [17].

This study aimed to investigate (i) whether conessine, a steroidal alkaloid compound, could act as a resistance modifying agent against multidrug-resistant Pseudomonas aeruginosa, and (ii) whether MexAB-OprM efflux pump is involved in the mechanism.

\section{Methods \\ Bacterial strains}

P. aeruginosa PAO1 strain K767 (wild-type), MexABOprM overexpressed strain K1455 (PAO1-nalB), and MexB deletion strain K1523 (PAO1- $\Delta$ mexB) were generously provided by Professor Dr. R. Keith Poole, Queen's University, Kingston, Ontario, Canada.

\section{Chemicals and media}

Phenylalanine-arginine $\beta$-naphthylamide (PA $\beta \mathrm{N}), 1-N$-phenylnaphthylamine (NPN), Hoechst 33,342 (H33342), conessine, and antibiotics were purchased from Sigma-Aldrich (St Louis, MO, USA). Dimethylsulfoxide (DMSO) and ethylenediaminetetraacetic acid (EDTA) were obtained from Merck (Merck, Germany).

Mueller-Hinton broth (MHB) and Tryptic soy agar (TSA) were purchased from Becton Dickinson Microbiology Systems (Sparks, MD, USA).

\section{Antibacterial activity assays}

Minimum inhibitory concentration was tested by broth microdilution assay in accordance with the Clinical and Laboratory Standards Institute (CLSI) recommendation [18]. Antibiotics used in this study were selected based on substrate specificity of Ade efflux pump in A. baumannii: cefotaxime for AdeDE pump, novobiocin for AdeIJK pump, rifampicin for AdeDE and AdeIJK pump, erythromycin, levofloxacin, and tetracycline for AdeABC, AdeDE, and AdeIJK pump. In addition, cefotaxime, levofloxacin, novobiocin, and tetracycline have been reported as substrates for MexAB-OprM in P. aeruginosa. Stock solution of novobiocin $(50 \mathrm{mg} / \mathrm{L})$, rifampicin $(1 \mathrm{mg} / \mathrm{L})$, levofloxacin (18 mg/L), erythromycin (2 mg/L), cefotaxime $(10 \mathrm{mg} / \mathrm{L})$, and $\mathrm{PA} \beta \mathrm{N}(10 \mathrm{mg} / \mathrm{L})$ were prepared in sterile deionized 
water. Tetracycline $(4 \mathrm{mg} / \mathrm{L})$ and conessine $(1 \mathrm{mg} / \mathrm{L})$ were dissolved in 95\% ethanol and 100\%DMSO, respectively. Serial dilutions of conessine, PA $\beta N$, and antibiotics were prepared in MHB. In order to investigate the effect of each agent, $100 \mu \mathrm{L}$ bacterial culture $\left(1 \times 10^{6} \mathrm{cfu} / \mathrm{mL}\right)$ was mixed with $100 \mu \mathrm{L}$ each conessine, PA $\beta \mathrm{N}$, or antibiotics. Synergistic effects of conessine $(20 \mathrm{mg} / \mathrm{L})$ or $\mathrm{PA} \beta \mathrm{N}$ $(25 \mathrm{mg} / \mathrm{L})$ and antibiotics were assessed using checkerboard assay by adding $100 \mu \mathrm{L}$ culture into a well containing $50 \mu \mathrm{L}$ conessine or PAßN and $50 \mu \mathrm{L}$ antibiotics. DMSO at a final concentration of $1 \%$ used as a negative control and PA $\beta \mathrm{N}$, an efflux pump inhibitor was used as a positive control. Plates were then read after $18 \mathrm{~h}$ of incubation at $37^{\circ} \mathrm{C}$. Each assay with three biological triplicates was repeated at least twice. A 4-fold or greater reduction in MIC values after addition of conessine or PA $\beta \mathrm{N}$ was considered significant. Fractional inhibitory concentration index (FICI) value was calculated for each combination according to the following formula [19]: $\mathrm{FICI}=(\mathrm{MIC}$ of efflux pump inhibitors in combination/MIC of efflux pump inhibitors alone) + (MIC of antibiotics in combination/MIC of antibiotics alone). Synergy, additivity, and antagonism were defined as FICI $<1,=1$, and $>1$, respectively.

\section{H33342 accumulation assay}

H33342 accumulation assay was performed to evaluate the effect of efflux pump inhibitors on the activity of MexAB-OprM efflux pump [20]. Briefly, overnight bacterial cultures were inoculated into MHB and rotated at $250 \mathrm{rpm}$ at $37^{\circ} \mathrm{C}$ for $4-5 \mathrm{~h}$. Bacterial cells were harvested by centrifugation (3000 rpm for $15 \mathrm{~min}$ ) and the cells were washed with phosphatebuffered saline containing $1 \mathrm{mM}$ $\mathrm{MgSO}_{4}$ and $20 \mathrm{mM}$ glucose. After centrifugation, the pellets were resuspended in the same buffer and $\mathrm{OD}_{600}$ of each suspension was adjusted to 0.4 . An aliquot of $100 \mu \mathrm{L}$ of the bacterial suspension was added into a well in black microtiter plate containing each of $50 \mu \mathrm{L}$ conessine $(20 \mathrm{mg} / \mathrm{L})$ or an efflux pump inhibitor, PA $\beta \mathrm{N}(25 \mathrm{mg} / \mathrm{L})$.

The final concentration of DMSO in all assays was $\leq 1 \%$. Plates were incubated at $37{ }^{\circ} \mathrm{C}$ for $15 \mathrm{~min}$ and $50 \mu \mathrm{L}$ H33342 $(2.5 \mu \mathrm{M})$ was added to each assay well. Fluorescence (excitation $355 \mathrm{~nm}$, emission $460 \mathrm{~nm}$ ) was measured at $37^{\circ} \mathrm{C}$ every $2.30 \mathrm{~min}$ for $1 \mathrm{~h}$ using a Varioskan Flash spectral scanning multimode reader. Each assay was repeated at least twice. Differences in accumulation in the presence of efflux pump inhibitors compared with the absence of efflux pump inhibitors were analysed for statistical significance using Student's $t$-test. $P$ value $\leq 0.05$ was considered significant.

\section{NPN uptake assay}

Ability of conessine to permeabilize $P$. aeruginosa outer membrane was assessed by NPN uptake assay [21]. NPN, an uncharged lipophilic molecule, fluoresces weakly in aqueous environments but becomes strongly fluorescent in nonpolar environments such as cell membranes. Briefly, overnight bacterial cultures were inoculated into MHB and rotated at $250 \mathrm{rpm}$ at $37{ }^{\circ} \mathrm{C}$ for $4-5 \mathrm{~h}$. Bacterial cells were harvested at $3000 \mathrm{rpm}$ for $15 \mathrm{~min}$, washed with $100 \mathrm{mM} \mathrm{NaCl}$ and $50 \mathrm{mM}$ sodium phosphate buffer (pH 7.0), and resuspended in the same buffer at $\mathrm{A}$ $600=0.1$ in the presence of $0.05 \%$ of glucose. An aliquot of $100 \mu \mathrm{L}$ of the bacterial suspension was added into a well in black microtiter plate containing each of $50 \mu \mathrm{L}$ conessine $(20 \mathrm{mg} / \mathrm{L})$ or EDTA $(100 \mu \mathrm{M})$ as a permeabilizer followed by adding $50 \mu \mathrm{L}$ of NPN $(40 \mu \mathrm{M})$. The final concentration of DMSO in all assays was $\leq 1 \%$. NPN fluorescence intensity (excitation $322 \mathrm{~nm}$, emission $424 \mathrm{~nm}$ ) was monitored at $37^{\circ} \mathrm{C}$ after $2.30 \mathrm{~min}$ for $1 \mathrm{~h}$ using a Varioskan Flash spectral scanning multimode reader (Thermo Fisher Scientific, Finland). Each assay was repeated at least twice. Differences in accumulation in the presence of efflux pump inhibitors compared with the absence of efflux pump inhibitors were analysed for statistical significance using Student's $t$-test. $P$ value $\leq 0.05$ was considered significant.

\section{Results}

\section{Intrinsic antibacterial activity of conessine, PA $\beta N$, and} antibiotics

MICs of conessine and PA $\beta \mathrm{N}$ for $P$. aeruginosa wildtype strain K767 (PAO1), MexAB-OprM overexpressed strain K1455 (PAO1-nalB), and MexB deletion strain K1523 (PAO1- $\Delta$ mexB) were determined. Intrinsic MICs of conessine in all strains were $40 \mathrm{mg} / \mathrm{L}$ while MICs of $\mathrm{PA} \beta \mathrm{N}$ were between 512 and $1024 \mathrm{mg} / \mathrm{L}$. At a concentration required to reduce MICs of antibiotics by at least 4-fold in $P$. aeruginosa strains, conessine $(20 \mathrm{mg} / \mathrm{L})$ and $\mathrm{PA} \beta \mathrm{N}(25 \mathrm{mg} / \mathrm{L})$ alone did not affect growth rate in all strains (data not present).

Susceptibility of $P$. aeruginosa strains to a range of antibiotics is shown in Table 1. Overexpression of MexAB-OprM conferred resistance to cefotaxime, erythromycin, levofloxacin, novobiocin, and tetracycline, except rifampicin. The experiments supported earlier report that resistance to rifampicin may involve mutations in $r p o B$ gene rather than as a simple function of expression of efflux systems [22]. A mutant strain with MexB deletion displayed susceptibility to cefotaxime, levofloxacin, and novobiocin. The findings indicate that extrusion of cefotaxime, levofloxacin, and novobiocin was mainly specific to MexAB-OprM efflux system. On the other hand, resistance to erythromycin, rifampicin, and tetracycline may involve other efflux pump systems. Together, the results are consistent with previous studies on substrate specificity of Mex efflux systems in P. aeruginosa $[23,24]$. 
Table 1 Modulation of antibiotic resistance in Pseudomonas aeruginosa by conessine

\begin{tabular}{|c|c|c|c|c|c|c|}
\hline \multirow[t]{2}{*}{ Bacterial strain } & \multirow[t]{2}{*}{ Antibiotic } & \multicolumn{3}{|c|}{ Antibiotic Minimum inhibitory concentration (mg/L) with } & \multicolumn{2}{|c|}{ Fractional inhibitory concentration index ${ }^{\mathrm{b}}$} \\
\hline & & $\left.\overline{\text { No EPl }}\right|^{a}$ & Conessine $(20 \mathrm{mg} / \mathrm{L})$ & PAßN (25 mg/L) & Conessine & PAßN \\
\hline \multirow[t]{6}{*}{ K767 (PAO1) } & Cefotaxime & 8 & 1 & 4 & 0.63 & 0.52 \\
\hline & Erythromycin & 128 & 16 & 64 & 0.63 & 0.52 \\
\hline & Levofloxacin & 0.25 & 0.03 & 0.03 & 0.63 & 0.15 \\
\hline & Novobiocin & 1024 & 128 & 128 & 0.63 & 0.15 \\
\hline & Rifampicin & 16 & 2 & 2 & 0.63 & 0.15 \\
\hline & Tetracycline & 8 & 1 & 4 & 0.63 & 0.52 \\
\hline \multirow[t]{6}{*}{ K1455 (PAO1-nalB) } & Cefotaxime & 64 & 8 & 64 & 0.63 & 1.02 \\
\hline & Erythromycin & 256 & 32 & 64 & 0.63 & 0.27 \\
\hline & Levofloxacin & 2 & 0.25 & 0.25 & 0.63 & 0.15 \\
\hline & Novobiocin & $>2048$ & 256 & 256 & 0.63 & 0.15 \\
\hline & Rifampicin & 16 & 2 & 2 & 0.63 & 0.15 \\
\hline & Tetracycline & 64 & 8 & 64 & 0.63 & 1.02 \\
\hline \multirow[t]{6}{*}{ K1523 (PAO1-AmexB) } & Cefotaxime & 1 & 0.13 & 0.13 & 0.63 & 0.17 \\
\hline & Erythromycin & 128 & 16 & 64 & 0.63 & 0.55 \\
\hline & Levofloxacin & 0.06 & 0.01 & 0.01 & 0.63 & 0.17 \\
\hline & Novobiocin & 64 & 64 & 64 & 1.50 & 1.05 \\
\hline & Rifampicin & 16 & 2 & 2 & 0.63 & 0.17 \\
\hline & Tetracycline & 8 & 1 & 2 & 0.63 & 0.30 \\
\hline
\end{tabular}

A reduction of at least 4 -fold is indicated in bold

${ }^{a}$ EPI, Efflux pump inhibitor

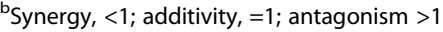

Efflux pump inhibitor activity of conessine in $P$. aeruginosa To verify that a mechanism by which conessine potentiate antibacterial activity was through inhibition of MexABOprM efflux, this study determined whether conessine enhanced antibacterial activity against wild-type, MexABOprM overexpressed, and MexB deletion strain. As shown in Table 1, antibiotic spectrum was affected by the addition of conessine and PA $\beta N$. Conessine significantly reduced MICs of all antibiotics by at least 8-fold in wildtype and MexAB-OprM overexpressed strain. In the overexpressed strain, the levels were comparable to those obtained in wild-type strain for cefotaxime, levofloxacin, and tetracycline. Interestingly, with erythromycin, novobiocin, and rifampicin, MICs were 4- to 8-fold less than those in the wild-type strain. With mexB deletion strain, conessine increased its susceptibility to almost all antibiotics, except novobiocin. Previous studies documented that other efflux pumps such as MexAB-OprM and MexCD-OprJ could export novobiocin [23, 24]. However, no synergistic effect was observed in MexB deletion strain, indicating that conessine may inhibit only MexAB-OprM pump to restore novobiocin activity. The results clearly demonstrated that conessine could be an inhibitor of MexAB-OprM efflux pump. Synergistic activity between other antibiotics (except novobiocin) and conessine observed in MexB deletion strain suggested that conessine might inhibit other efflux systems present in $P$. aeruginosa. Conessine increased susceptibility to rifampicin in all strains when this agent was not exported by MexABOprM efflux pump, indicating that conessine enhanced antibiotic susceptibility in MexAB-OprM overexpressed strain independent of an impact on MexAB-OprM. Restoration of rifampicin efficacy by conessine might result in increase in antibiotic susceptibility. In addition, conessine has the same 8 -fold reduction impact on antibiotic resistance in the wild-type, mexB deletion, and MexAB-OprM overexpressed strain, suggesting that conessine may act on other intrinsic resistance determinants.

A well-known inhibitor of RND efflux systems, PA $\beta N$ [5], increased susceptibility to all antibiotics in the wildtype strain whereas in MexAB-OprM overexpressed strain, the inhibitor could not reduce MICs of tetracycline and cefotaxime. In MexB deletion strain, MICs of all antibiotics except novobiocin were affected by PA $\beta \mathrm{N}$. The results are consistent with previous reports indicating that PA $\beta \mathrm{N}$ inhibited MexAB-OprM pump as well as other Mex efflux systems in $P$. aeruginosa such as MexCD-OprJ and MexEF-OprN [5, 25]. Remarkably, conessine could decrease MICs of levofloxacin, novobiocin, and rifampicin in all strains to the levels comparable 
to those when combined with PA $\mathrm{N}$. With cefotaxime, erythromycin, and tetracycline, conessine could lower the MICs in all the strains better than PA $\beta N$. The findings clearly demonstrated that conessine could reduce MIC against $P$. aeruginosa 2 - to 8 -fold lower magnitude in the tested strains, compared with PA $\beta \mathrm{N}$.

\section{Inhibition of efflux systems}

H33342 accumulation assay was used to confirm that conessine directly inhibited efflux pump systems in $P$. aeruginosa [20]. Conessine at $20 \mathrm{mg} / \mathrm{L}$ demonstrated an increase in H33342 accumulation in a wild-type strain (Fig. 1a). However, the effect of conessine was less than PA $\beta N$. A similar pattern was observed from an overexpressed strain (Fig. 1b). In a pump-deficient strain (Fig. 1c), conessine could elevate the level of H33342 accumulation to the level comparable to those when combined with PA $\beta N$. The findings suggested that conessine inhibited MexAB-OprM efflux pump and other efflux pump systems in $P$. aeruginosa.
Interaction with outer membrane of $P$. aeruginosa

Ability of conessine to permeabilize outer membrane of $P$. aeruginosa was determined with intact cells by NPN uptake assay [21]. As shown in Fig. 2, conessine $(20 \mathrm{mg} /$ L) did not weaken outer membrane of all $P$. aeruginosa strains as evidenced by no increase in NPN uptake. Conversely, a positive control EDTA $(100 \mu \mathrm{M})$ promoted NPN uptake across outer membrane of all tested strains as indicated by a significant increase in NPN uptake. The findings suggested that conessine has no effect on outer membrane permeability. Different $P$. aeruginosa genotypes exhibited different level of fluorescence especially in MexB deletion strain which demonstrated the highest level of fluorescence. Regarding MexAB-OprM overexpressed strain, the level of fluorescence was lower than in wild-type due to the efflux pump activity of MexAB-OprM. Conessine did not increase NPN uptake in all the tested strains, thus fluorescence level after the bacterial cells treated with conessine were the same. The level of fluorescence in the presence of conessine did

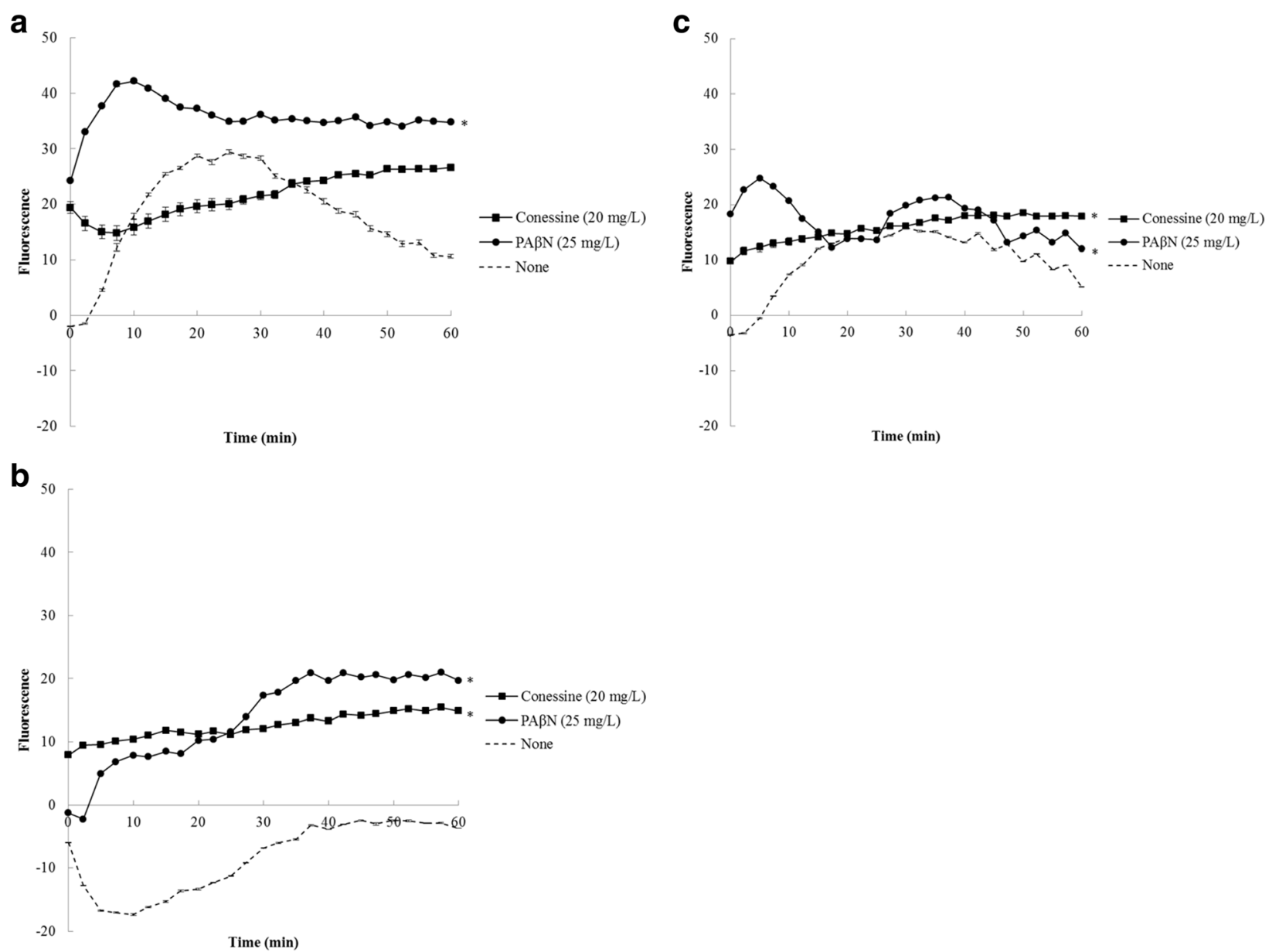

Fig. 1 Intracellular concentration of Hoechst 33,342 in the presence of conessine (20 mg/L) and phenylalanine-arginine $\beta$-naphthylamide (PABN) (25 mg/L) in Pseudomonas aeruginosa K767 (PAO1) (a), P. aeruginosa K1455 (PAO1-nalB) (b), and P. aeruginosa K1523 (PAO1- $\triangle$ mexB) (c). Data were shown as average of two independent experiments. Error bars displayed \pm SEM and * indicated significant $(P$ value $\leq 0.05)$ 


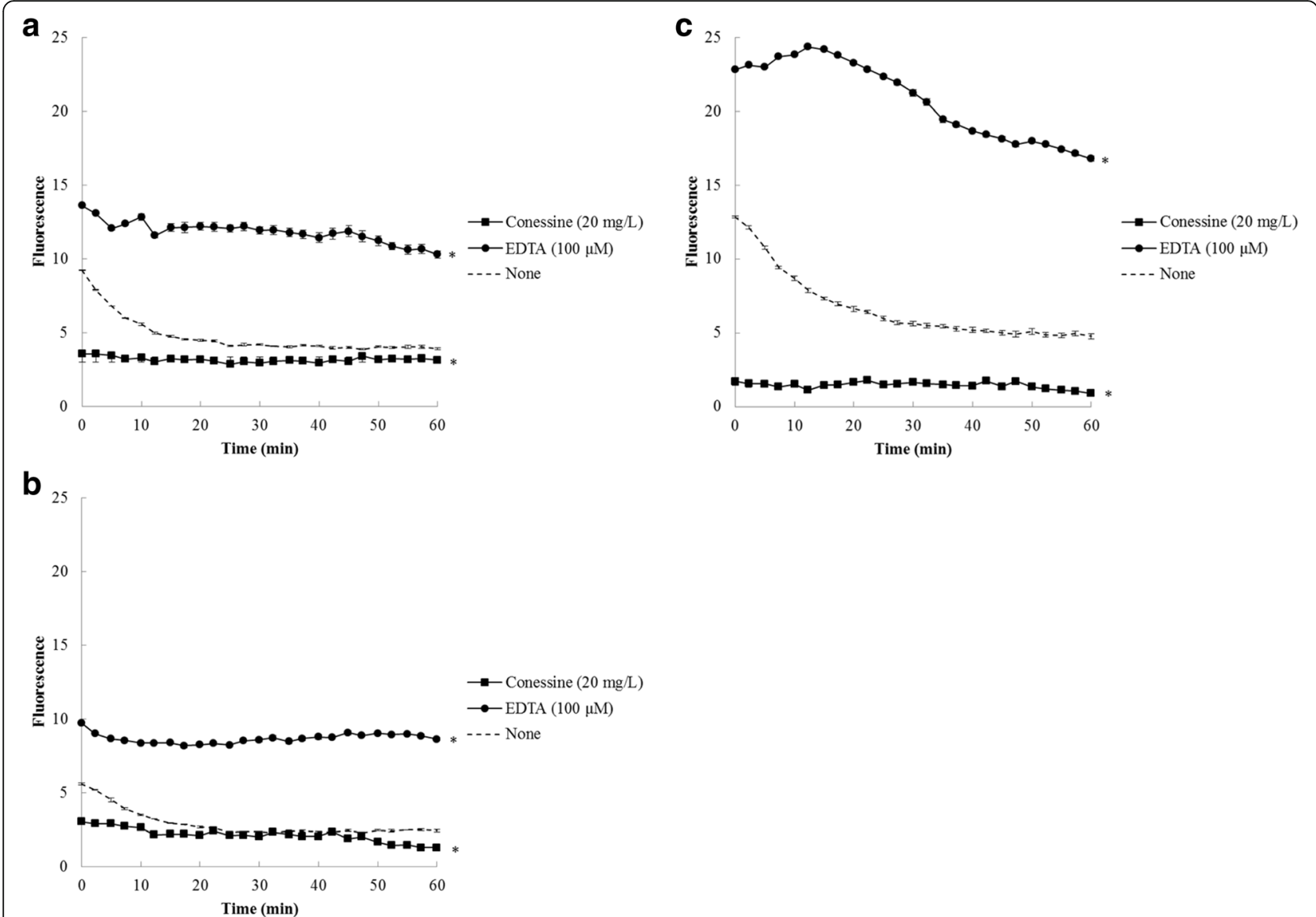

Fig. 2 Intracellular concentration of 1-N-phenylnaphthylamine in the presence of conessine (20 mg/L) and ethylenediaminetetraacetic acid (EDTA) $(100 \mu \mathrm{M})$ in Pseudomonas aeruginosa K767 (PAO1) (a), P. aeruginosa K1455 (PAO1-nalB) (b), and P. aeruginosa K1523 (PAO1- $\triangle$ mexB) (c). Data were shown as average of two independent experiments. Error bars displayed \pm SEM and ${ }^{*}$ indicated significant $(P$ value $\leq 0.05)$

not lower than the control, in particular for a pumpdeficient strain (Fig. 2c) but because of the increase in fluorescence in the deletion strain.

\section{Discussion}

Inhibition of MexAB-OprM efflux pump appears to be an attractive approach to restore efficacy of antibiotics that were substrates of this pump. Herein, we describe efficacy of conessine, an inhibitor of RND class MexABOprM efflux pump, which is the major efflux pump and plays a vital role in MDR phenotype in $P$. aeruginosa. The present study demonstrated that conessine displayed characteristics of an efflux pump inhibitor as previously documented by Lomovskaya et al. [5].

A mechanism of efflux pump inhibition by conessine was possibly through competitive inhibition and/or blockage of access to the substrate binding site of MexB. In comparison with MexB-specific PA $\beta \mathrm{N}$, the compounds might interact with "G-loop" or "switch loop", which separates the distal and the proximal binding sites. G-loop has been proposed to be involved in movement of substrates from the proximal to the distal site. Therefore, efflux pump inhibitors inhibited MexB extrusion of various substrates through binding to G-loop [26]. Differences in spectrum of antibiotics enhanced by conessine versus $\mathrm{PA} \beta \mathrm{N}$ suggested that conessine may bind to a different site in MexB binding pocket.

\section{Conclusion}

Conessine potentiated antibiotic activity by inhibiting MexAB-OprM efflux pump in $P$. aeruginosa. The findings revealed that a resistance modifying agent, conessine may be employed as a novel inhibitor for the alternative therapy against multidrug-resistant $P$. aeruginosa.

\section{Abbreviations}

CCCP: Carbonyl cyanide m-chlorophenylhydrazone; CLSI: Clinical and Laboratory Standards Institute; DMSO: Dimethylsulfoxide;

EDTA: Ethylenediaminetetraacetic acid; EPI: Efflux pump inhibitor;

FICl: Fractional inhibitory concentration index; H33342: Hoechst 33342;

MDR: Multidrug resistant; MHB: Mueller hinton broth; MHB: Mueller-Hinton broth; MIC: Minimal inhibitory concentration; NMP: 1-(1-Naphthylmethyl)piperazine; NPN: 1-N-phenylnaphthylamine; PABN: Phenylalanyl arginyl $\beta$ - 
naphthylamide; PMBN: Polymyxin B nonapeptide; RND: Resistancenodulation-division; TSB: Tryptic soy broth

\section{Acknowledgements}

Not applicable.

\section{Funding}

This work was supported by the Royal Golden Jubilee Ph.D. Program

(Grant No. PHD/0041/2556) and TRF Senior Research Scholar

(Grant No. RTA5880005), the Thailand Research Fund.

\section{Availability of data and materials}

The data and materials are included within the article.

\section{Authors' contributions}

TS performed experiments and wrote the first draft of a manuscript. BY and CS helped in extraction of alkaloids. PS and SPV supervised parts of the experimental work. SPV edited the manuscript. All authors read and approved the final manuscript.

\section{Ethics approval and consent to participate}

Not applicable.

\section{Consent for publication}

Not applicable.

\section{Competing interests}

The authors declare that they have no competing interests.

\section{Publisher's Note}

Springer Nature remains neutral with regard to jurisdictional claims in published maps and institutional affiliations.

\section{Author details}

${ }^{1}$ Department of Microbiology and Excellence Research Laboratory on Natural Products, Faculty of Science and Natural Product Research Center of Excellence, Prince of Songkla University, Hat Yai, Songkhla 90112, Thailand. ${ }^{2}$ Graduate Program, Faculty of Allied Health Sciences, Thammasat University, Pathumthani 12121, Thailand. ${ }^{3}$ Faculty of Traditional Thai Medicine and Excellence Research Laboratory on Natural Products, Faculty of Science and Natural Product Research Center of Excellence, Prince of Songkla University, Hat Yai, Songkhla 90112, Thailand. ${ }^{4}$ Department of Chemistry and Center for Innovation in Chemistry, Faculty of Science, Ramkhamhaeng University, Bangkapi, Bangkok 10240, Thailand. ${ }^{5}$ Department of Biomedical Sciences, Faculty of Medicine, Prince of Songkla University, Hat Yai, Songkhla 90112, Thailand.

Received: 24 October 2016 Accepted: 7 August 2017 Published online: 14 August 2017

\section{References}

1. Hirsch EB, Tam VH. Impact of multidrug-resistant Pseudomonas aeruginosa infection on patient outcomes. Expert Rev Pharmacoecon Outcomes Res. 2010;10:441-51.

2. Poole K. Pseudomonas Aeruginosa: resistance to the max. Front Microbiol. 2011;2:65

3. Poole K. Multidrug efflux pumps and antimicrobial resistance in Pseudomonas aeruginosa and related organisms. J Mol Microbiol Biotechnol. 2001;3:255-64.

4. Saito K, Yoneyama H, Nakae T. nalB-type mutations causing the overexpression of the MexAB-OprM efflux pump are located in the mexR gene of the Pseudomonas aeruginosa chromosome. FEMS Microbiol Lett. 1999;179:67-72.

5. Lomovskaya O, Warren MS, Lee A, Galazzo J, Fronko R, Lee M, et al. Identification and characterization of inhibitors of multidrug resistance efflux pumps in Pseudomonas aeruginosa: novel agents for combination therapy. Antimicrob Agents Chemother. 2001;45:105-16.

6. Pages JM, Masi M, Barbe J. Inhibitors of efflux pumps in gram-negative bacteria. Trends Mol Med. 2005;11:382-9.

7. Gill EE, Franco OL, Hancock RE. Antibiotic adjuvants: diverse strategies for controlling drug-resistant pathogens. Chem Biol Drug Des. 2015;85:56-78.
8. Lomovskaya O, Bostian KA. Practical applications and feasibility of efflux pump inhibitors in the clinic-a vision for applied use. Biochem Pharmacol. 2006;71:910-8.

9. Abreu AC, McBain AJ, Simoes M. Plants as sources of new antimicrobials and resistance-modifying agents. Nat Prod Rep. 2012;29:1007-21.

10. Negi N, Prakash P, Gupta ML, Mohapatra TM. Possible role of curcumin as an efflux pump inhibitor in multi drug resistant clinical isolates of Pseudomonas aeruginosa. J Clin Diagn Res. 2014;8:DC04-7.

11. Chusri S, Siriyong T, Na-Phatthalung P, Voravuthikunchai SP. Synergistic effects of ethnomedicinal plants of Apocynaceae Family and antibiotics against clinical isolates of Acinetobacter baumannii. Asian Pac J Trop Med. 2014;7:456-61.

12. Phatthalung PN, Chusri S, Voravuthikunchai SP. Thai ethnomedicinal plants as resistant modifying agents for combating Acinetobacter baumannii infections. BMC Complement Altern Med. 2012:12:56.

13. Chusri S, Na-Phatthalung P, Siriyong T, Paosen S, Voravuthikunchai SP. Holarrhena antidysenterica as a resistance modifying agent against Acinetobacter baumannii: its effects on bacterial outer membrane permeability and efflux pumps. Microbiol Res. 2014;169:417-24.

14. Siriyong $T$, Chusri $S$, Srimanote $P$, Tipmanee V, Voravuthikunchai SP. Holarrhena antidysenterica extract and its steroidal alkaloid, conessine, as resistance modifying agents against extensively drug-resistant Acinetobacter baumannii. Microb Drug Resist. 2016;22:273-82.

15. Damier-Piolle L, Magnet S, Brémont S, Lambert T, Courvalin P. AdelJK, a resistance-nodulation-cell division pump effluxing multiple antibiotics in Acinetobacter baumannii. Antimicrob Agents Chemother. 2008;52:557-62.

16. Sinha S, Sharma A, Reddy PH, Rathi B, Prasad NVSRK, Vashishtha A. Evaluation of phytochemical and pharmacological aspects of Holarrhena antidysenterica (Wall.): a comprehensive review. J Pharm Res. 2013;6:488-92.

17. Kumar N, Singh B, Bhandari P, Gupta AP, Kaul VK. Steroidal alkaloids from Holarrhena antidysenterica (L.) WALL. Chem Pharm Bull. 2007:55:912-4.

18. National Committee for Clinical Laboratory Standards (NCCLS). Methods for Dilution Antimicrobial Susceptibility Tests for Bacteria That Grow AerobicallyNinth Edition: Approved Standard M07-A9. Wayne, PA, USA. 2012.

19. Berenbaum MC. A method for testing for synergy with any number of agents. J Infect Dis. 1978;137:122-30.

20. Coldham NG, Webber M, Woodward MJ, Piddock LJ. A 96-well plate fluorescence assay for assessment of cellular permeability and active efflux in Salmonella enterica serovar Typhimurium and Escherichia coli. J Antimicrob Chemother. 2010;65:1655-63.

21. Ocaktan A, Yoneyama $H$, Nakae T. Use of fluorescence probes to monitor function of the subunit proteins of the MexA-MexB-oprM drug extrusion machinery in Pseudomonas aeruginosa. J Biol Chem. 1997;272:21964-9.

22. Mariam DH, Mengistu $Y$, Hoffner SE, Andersson DI. Effect of rpoB mutations conferring rifampin resistance on fitness of Mycobacterium tuberculosis. Antimicrob Agents Chemother. 2004:48:1289-94.

23. Lister PD, Wolter DJ, Hanson ND. Antibacterial-resistant Pseudomonas aeruginosa: clinical impact and complex regulation of chromosomally encoded resistance mechanisms. Clin Microbiol Rev. 2009;22:582-610.

24. Masuda N, Sakagawa E, Ohya S, Gotoh N, Tsujimoto H, Nishino T. Substrate specificities of MexAB-OprM, MexCD-OprJ, and MexXY-OprM efflux pumps in Pseudomonas aeruginosa. Antimicrob Agents Chemother. 2000;44:3322-7.

25. Renau TE, Leger R, Flamme EM, Sangalang J, She MW, Yen R, et al. Inhibitors of efflux pumps in Pseudomonas aeruginosa potentiate the activity of the fluoroquinolone antibacterial levofloxacin. J Med Chem. 1999:42:4928-31.

26. Opperman TJ, Nguyen ST. Recent advances toward a molecular mechanism of efflux pump inhibition. Front Microbiol. 2015:6:421. 